\title{
The effect of a sit-stand workstation intervention on daily sitting, standing and physical activity: protocol for a 12 month workplace randomised control trial
}

Jennifer Hall ${ }^{1 *}$, Louise Mansfield ${ }^{1,2}$, Tess Kay ${ }^{1,2}$ and Alison K McConnell ${ }^{1}$

\begin{abstract}
Background: A lack of physical activity and excessive sitting can contribute to poor physical health and wellbeing. The high percentage of the UK adult population in employment, and the prolonged sitting associated with desk-based office-work, make these workplaces an appropriate setting for interventions to reduce sedentary behaviour and increase physical activity. This pilot study aims to determine the effect of an office-based sit-stand workstation intervention, compared with usual desk use, on daily sitting, standing and physical activity, and to examine the factors that underlie sitting, standing and physical activity, within and outside, the workplace.

Methods/Design: A randomised control trial (RCT) comparing the effects of a sit-stand workstation only and a multi-component sit-stand workstation intervention, with usual desk-based working practice (no sit-stand workstation) will be conducted with office workers across two organisations, over a 12 month period $(\mathrm{N}=30)$. The multicomponent intervention will comprise organisational, environmental and individual elements. Objective data will be collected at baseline, and after 2-weeks, 3-months, 6-months and 12-months of the intervention. Objective measures of sitting, standing, and physical activity will be made concurrently (ActivPAL3 ${ }^{\mathrm{TM}}$ and ActiGraph (GT3X+)). Activity diaries, ethnographic participant observation, and interviews with participants and key organisational personnel will be used to elicit understanding of the influence of organisational culture on sitting, standing and physical activity behaviour in the workplace.
\end{abstract}

Discussion: This study will be the first long-term sit-stand workstation intervention study utilising an RCT design, and incorporating a comprehensive process evaluation. The study will generate an understanding of the factors that encourage and restrict successful implementation of sit-stand workstation interventions, and will help inform future occupational wellbeing policy and practice. Other strengths include the objective measurement of physical activity during both work and non-work hours.

Trail registration: Clinicaltrials.gov identifier NCT02172599, 22nd June 2014.

Keywords: Health, Multi-component intervention, Physical activity, Process evaluation, Randomised control trial, Sedentary behaviour, Sit-stand workstation, Sitting, Standing, Workplace

\footnotetext{
* Correspondence: Jennifer.Hall@brunel.ac.uk

'Division of Sport, Health and Exercise Sciences, Department of Life Sciences,

Brunel University London, London, Middlesex UB8 3PH, England, UK

Full list of author information is available at the end of the article
} 


\section{Background}

Sedentary behaviour (taken from the Latin term sedere; to sit) has been operationally defined as any activity that involves an energy expenditure of $\leq 1.5$ METS (metabolic equivalents), performed in seated or lying position [1]. Emerging research evidence identifies sedentary behaviour as a risk factor for negative health outcomes in the adult population; this risk is distinct and independent from risk associated with physical inactivity [2-4]. A lack of breaks in sedentary time is also related to metabolic disease risk [5,6]. For example, lipoprotein lipase is regulated differently following sedentary behaviour and physical activity; whilst a reduction in LPL activity occurs only in oxidative muscle fibres following sedentary behaviour, physical activity promotes increased LPL activity in glycolytic muscle fibres [7]. Further, a recent intervention study found that self-reported sitting time was significantly associated with a component of DNA; telomere length [8] Shortening of telomeres is associated with ageing and onset of disease [9]. This evolving evidence raises the question, "What should the person who sleeps an average of 8 hours per day [and is physically active for 30 minutes per day] do for the remaining 15.5 hours?" [10]. Thus, research and policy guidelines, such as the UK's 'Start Active, Stay Active' policy are now promoting both increases in physical activity and decreases in sedentary behaviour, stating adults should "minimise the amount of time spent being sedentary (sitting) for extended periods" [11].

Physical activity has been defined as "any bodily movement...that results in energy expenditure" [12]. There is a well-established body of evidence supporting the health benefits of physical activity [13]. However, physical activity is a complex behaviour; participation differs among different socio-economic groups and in the contexts and environments that contribute to health inequalities [14-16]. In the UK, it is estimated that over $73 \%$ of working age adults were engaged in full time or part time employment between April 2014 and June 2014 [17] and that adults spend an average of $60 \%$ of the working day at the workplace [18]. Thus, the workplace is an ideal setting for promoting physical activity and reductions in sitting. The office is a particularly important workplace setting, given the high proportion of sedentary time in desk-based office workers. For example, an observational study measured objectively the sedentary behaviour and physical activity of 50 office-based employees for 7 consecutive days; it found that, on average, $81.8 \%$ of working hours were spent engaged in sedentary behaviours, compared to $68.9 \%$ of non-work hours. Additionally, there were fewer breaks in sedentary time, and less light-intensity activity, during working hours than during non-working hours $(\mathrm{p}<0.001)$ [19]. This suggests that office-workers are more sedentary and less active during work than outside work and thus the office-based workplace is a crucial context for intervention to promote increases in physical activity and reductions in prolonged sitting. In desk-based office occupations, prolonged sitting is likely to occur in the context of travelling to work and participating in meetings, however, approximately twothirds of workplace sitting time is spent at a desk [20].

One response to reducing sitting time in the workplace is the installation of sit-stand workstations. Sit-stand workstations offer height-adjustable desk equipment for computer screens and keyboards allowing employees a choice of desk-based working positions. Several studies have examined the contribution of sit-stand workstations to a range of sedentary behaviour, health and productivity outcomes. Previous sit-stand workstation intervention studies have seen varying reductions in the amount of time spent sitting, ranging from 66 minutes to 143 minutes per day [21]. A recent meta-analysis of activity-permissive workstations revealed that the reduction in sedentary behaviour is greater in interventions that incorporate a change to the working environment (i.e. an activitypermissive workstation), than interventions that do not [22]. Physiological research shows that using a sit-stand workstation for 185 minutes, immediately after eating, can reduce post-prandial glucose excursion by $43 \%$ [23]. A recent review of empirical studies examining the relationship between sit-stand workstations and a range of measures of productivity revealed that whilst the majority of studies show that using a sit-stand workstation had no influence on productivity, no studies showed a reduction in productivity [24]. In line with an ecological approach to behaviour change [25], initial research has shown that greater reductions in sitting time $(56 \mathrm{~min} / 8$-hour workday) can be achieved as a result of a multi-component sitstand workstation intervention (incorporating individual and organisational level components) compared to receiving a sit-stand workstation alone [26]. However, this needs to be replicated over a prolonged time-period (e.g. 12 months), on outcomes related to physical activity (distinct from sitting, standing and stepping). Whilst the feasibility of using sit-stand workstations has been qualitatively explored with desk-based office workers [27], outcomefocused studies dominate this research area. Understanding of the social processes that encourage and restrict standing and physical activity in the workplace is required. The absence of systematic and rigorous process evaluations of sit-stand workstation interventions will impede the wider adoption of sit-stand workstations across organisations $[28,29]$.

\section{Aims}

The primary aim is to determine the effect of a 12 month multi-component sit-stand workstation intervention, incorporating organisational, environmental and individual 
level strategies, on physical activity within the workplace (primary outcome). Secondary aims are to: determine the effect of the sit-stand workstation intervention on physical activity outside of the workplace (secondary outcome), and to determine whether the multi-component intervention is more effective than the sit-stand workstation provision alone. The process evaluation will examine the factors that influence sitting, standing and active behaviours in the workplace.

\section{Methods/Design}

\section{Study design}

An RCT comparing the effects of a sit-stand workstation only and a multi-component sit-stand workstation intervention, with usual office-based working practice (no sitstand workstation) will be implemented. The study will be conducted with office workers across two organisations according to Cochrane recommendations [30], with 'individuals' being the unit of randomisation. The design employs two intervention arms: (1) a multi-component sit-stand workstation intervention (SS-MC); and (2) sit-stand workstation only (SS-O). A control arm (CG) for usual deskbased working practice (no sit-stand workstation) will also be included. Objective data collection will take place at 5 time-points over the course of 12-months: baseline (approximately one month before desk installation), and then post-installation at 2-weeks, 3-months, 6-months and 12months. See Figure 1 for an overview of the study design and major study components. Ethics approval has been granted by Brunel University London local research ethics committee. All participants will receive a participant information sheet, which details their ethical rights, and provide written informed consent. The conduct of this study will follow the CONSORT guidelines (http://www. consort-statement.org/).

\section{Recruitment \\ Recruitment of organisations and study sites}

Two organisations, Macmillan Cancer Support and Public Health England (PHE) will provide use of workplaces (one per organisation) and employees as participants for the study. Macmillan Cancer Support is a charitable organisation that provides support for people living with and affected by cancer. The UK Office based in Central London, UK is the worksite selected for this study. Public Health England is a governmental organisation responsible for protecting and improving the nation's health and wellbeing, and reducing health inequalities. One PHE worksite in central London will be used in this study.

\section{Recruitment of participants}

Participants will be recruited via internal advertisement. At PHE, this will involve an email sent to all employees at the chosen worksite. At Macmillan Cancer Support, this involves an email sent to all employees at the chosen worksite, alongside their standard internal communication channels (Yammer page, posters). All potential participants will be required to attend a 45-60 minute recruitment workshop at their organisation. If they are unable to attend a workshop, they must engage in a 30 minute telephone conversation with the lead researcher to discuss the study requirements to be eligible to formally apply to take part in the research.

Potential participants will be invited to complete an expression of interest form that asks questions regarding age, gender, ethnicity, and disability status, as well as what interests them about sit-stand workstations, and whether they, or a close friend or family member, have ever had a cancer diagnosis*.

"Funding acquired for this study requires that some of the participants have been affected by cancer; via a personal cancer diagnosis and/or a close friend or family member being diagnosed with cancer.

\section{Eligibility criteria}

Participants must be full-time employees on a permanent or fixed term contract until the anticipated study end date, with no plans to leave the organisation, or be absent for an extended period ( $\geq 4$ weeks). Participants must engage primarily in desk-based office-work, be present at the worksite $\geq 4$ days a week, and must be at least 18 years of age. Participants must not have engaged in standing-based desk work in the four weeks prior to baseline data collection. Participants must not have been advised to avoid prolonged standing by a health professional; or be unable to stand.

Certain work teams at one of the participating organisations are excluded owing to the sensitivity of their work and current desk configuration; the current desks would need to be significantly adapted to accommodate the sit-stand equipment.

\section{Participant selection}

Of the potential participants that attend a recruitment workshop and complete the expression of interest form, those that do not meet the eligibility criteria will be excluded immediately. Thirty participants will then be selected strategically to take part in the research; selection will ensure the sample is representative of the range of characteristics on the expression of interest form (see above). The participants that are not selected will be put on a waiting list in case of drop-outs.

\section{Randomisation}

Participants will be allocated randomly to one of three arms using an online randomisation programme (www. randomizer.org). Participants from the two participating 


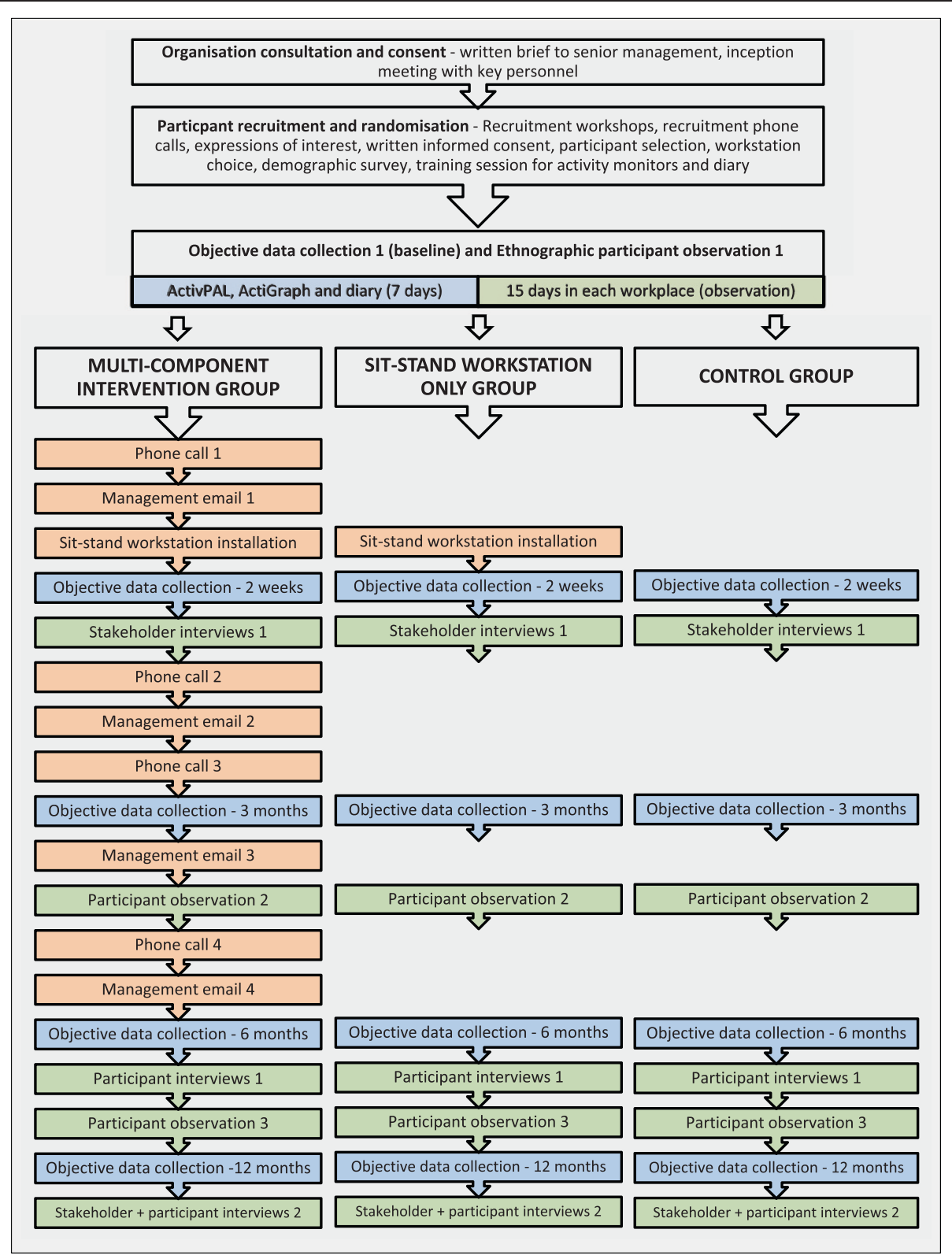

Figure 1 Study overview including recruitment and study design, intervention procedures and data collection.

organisations will be randomised separately, to achieve an equal number in each study arm in both organisations. Given the nature of the intervention, i.e. the fact that participants may or may not receive a sit-stand workstation, it will not be possible to blind participants or researchers to arm allocation. However, concealment allocation will be implemented [27].

\section{Trial status}

At the date of manuscript submission participant recruitment has been completed, and data collection has commenced but has not been completed.

\section{Sit-stand multi-component intervention}

\section{Theoretical basis and intervention development}

The intervention to be delivered to the SS-MC arm aligns with the World Health Authority's promotion of a healthy workplace model [31]. This theoretical framework emphasises that best-practice workplace health interventions should involve an integrated approach involving organisation and individual level approaches to behaviour change [32]. This is based on an ecological definition of health and approach to behaviour change, which recognises that many factors influence health behaviour and that psychosocial influences on physical 
activity and sedentary behaviour vary in different contexts $[25,33]$. The content of the intervention will be based on previous interventions in a similar context e.g. $[26,34,35]$ and will incorporate behaviour change techniques, including motivational interviewing [36,37]. The multi-component intervention will be refined to reflect the specific context (based on discussion with the respective organisation) and employee's needs (following the 'personalised inception conversation', see below) [38]. The intervention will take effect for the first six-months following the installation of sit-stand workstations.

\section{Multi-component intervention procedures}

The multi-component intervention comprises organisational, environmental, and individual level elements, in accordance with the healthy workplace model [31]. For an overview of the intervention procedures within the overall context of the study please see Figure 1.

Organisational level procedures These procedures aim to enhance the participants' perceived level of organisational support for the sit-stand workstation intervention. It will consist of four emails to participants in the SS-MC arm from organisational managers who are responsible for workplace wellbeing. These emails will be sent one week before the installation of the workstation, and at weeks 6,14 and 24 following the installation of the workstations. Emails will provide information on (a) explicit organisational support for using sit-stand workstations (motivational information) (b) the negative health impacts of sitting at work, and (c) strategies to reduce sitting and increase standing in the workplace.

Environmental level procedures The environmental element will involve changes to the physical environment that are expected to facilitate reduced sitting and increased physical activity. A sit-stand workstation, i.e. height-adjustable desk equipment allowing employees a choice of desk-based working positions will be provided to participants both the SS-O and SS-MC arms for 12-months. Participants will have the choice between two models of workstation (Ergotron Workfit-A or Workfit-D, www.ergotron.com). The lead researcher will explain the differences between the two workstations at the recruitment workshop, a flyer will be given to participants, and they will have the opportunity to 'test out' a demo workstation within their organisation before they choose their preferred workstation. Participants will receive written and verbal instruction on the correct ergonomic posture for both sitting and standing, upon installation of the workstations.

Individual level procedures Participants in the SS-MC arm will receive four 5-10 minute telephone calls (before installation of the sit-stand workstation, and at weeks 3, 10 and 19) from the lead researcher who is experienced in motivational interviewing, as a method for "enhancing intrinsic motivation to change, by exploring and resolving ambivalence" [39]. These telephone calls will engage participants in conversations related to the sit-stand workstations and physical activity that will follow the principles of engaging, guiding and evoking to motivate and support participants to reduce their inactivity and increase activity, primarily at the workplace.

\section{Data collection}

The research design is underpinned by the standard evaluation framework for physical activity programmes, incorporating both outcome and process evaluation data collection [40]. See Figure 1 for an overview of the data collection procedures.

\section{Participant profile data}

Profile data will be collected, through the expression of interest form (detailed earlier) and a short survey undertaken at a monitoring training session, before the installation of the sit-stand workstations. Questions on the short survey will include participants' height, weight, job title, household composition, personal monthly income, level of education, and sexuality.

\section{Outcome data}

Primary outcomes are: daily time spent sitting, standing, in light and moderate-vigorous physical activity, the average number of sit-stand transitions per hour, number of prolonged sitting bouts $\geq 30 \mathrm{~min}$, and total physical activity energy expenditure during work hours. Secondary outcomes will be the same, but over the course of the entire day (see Table 1). These will be measured at baseline (approximately one month before sit-stand workstation

Table 1 Study outcome measures taken at baseline, 2 weeks, 3 months, 6 months and 12 months

\begin{tabular}{lcc}
\hline & Working hours & Total hours \\
\hline ActivPAL3 $^{\text {TM }}$ & & $\checkmark$ \\
\hline - Sitting time & $\checkmark$ & $\checkmark$ \\
- Number of prolonged ( $\geq 30$ min) & $\checkmark$ & $\checkmark$ \\
sitting bouts & & $\checkmark$ \\
- Number of sit-to-stand transitions & $\checkmark$ & \\
- Standing time & $\checkmark$ & $\checkmark$ \\
\hline ActiGraph (GT3X+) & & $\checkmark$ \\
- Time in light physical activity (min) & $\checkmark$ & $\checkmark$ \\
- Time in moderate physical activity (min) & $\checkmark$ & $\checkmark$ \\
- Time in vigorous physical activity (min) & $\checkmark$ & $\checkmark$ \\
- Time in MVPA in 10-min bouts (min) & $\checkmark$ & \\
- Counts per minute & $\checkmark$ & \\
\hline
\end{tabular}


installation) and four subsequent time-points following workstation installation; two-weeks, 3-months, 6-months and 12-months. Each data collection period will be 7-days. Sitting, standing and sit-stand transitions will be assessed using an ActivPAL3 ${ }^{\mathrm{ma}}$ micro monitor (PAL Technologies Limited, Glasgow, UK) and light, moderate and vigorous physical activity will be assessed using an accelerometer (ActiGraph GT3X+, ActiGraph, Pensacola, FL, USA). The ActivPAL $^{\text {mi }}$ provides accurate measures of sitting time and sit-to-stand transitions per hour and sit-stand transitions, when compared to direct observation in officebased free-living environments $[41,42]$. The ActivPAL $3^{\mathrm{m}}$ will be worn continuously on the centre of the right thigh, following insertion into a nitrile sleeve and wrapping in a waterproof dressing. Participants will be given extra materials to change the dressing when required. Participants will be requested to wear the monitor continuously for each 7-day data collection period. The ActiGraph GT3X+ is a tri-axial accelerometer that accurately classifies physical activity intensity in free-living environments [43]. It will be worn on the hip via an elastic belt during waking hours only, excluding time spent engaged in water-based activities. Participants will be asked to record non-wear time of both monitors, and time spent at the workplace for all 7-day data collection periods.

\section{Process evaluation}

In alignment with the Medical Research Council guidelines on evaluating complex interventions [38], the RCT is accompanied by a systematic and rigorous process evaluation to gain an understanding of how sit-stand workstation interventions work in practice [44]. Process evaluations are particularly important in multi-site interventions to explore differences in implementation and outcomes [45].

Activity diaries Activity diaries will be used to determine the type and context of active and inactive behaviours, to facilitate understanding of the factors that influence sitting, standing and physical activity in the workplace context. Participants will be asked to record the behaviour that they spent the most time doing during each hour, during each 7-day objective data collection period. A text message, reminding participants to complete their activity diary, will be sent to participants once daily throughout each data collection period. Text message models have shown success in increasing compliance to data collection [46].

Ethnographic participant observation This method will be used to understand organisational culture, placing importance on the perceptions of the people under study within their usual environment [47]. The ethnographic participant observation will provide insight into the context within which the intervention is taking place; this will elicit understanding of the feasibility and acceptability of sit-stand workstation interventions, and the social processes that underpin use of the sit-stand workstations and physical activity in the workplace. The participant observation involves the lead researcher taking a volunteering role within the participating organisations. There will be three observation phases within each worksite; before the installation of sit-stand workstations, 4-5 months following the installation of sit-stand workstations, and 10-11 months following the installation of sit-stand workstations. The lead researcher will spend approximately 12 full working days at the workplace during each phase, engaging in work tasks related to physical activity and/or workplace health, set by the volunteering organisation.

Key stakeholder and participant interviews Qualitative interviews will enable explorations of perceptions and meaning to increase understanding of a particular phenomenon [48]. Thus, interviews will be used within this study to delineate the perceptions of both key stakeholders and participants in relation to sit-stand working and physical activity in the workplace, to understand the factors that influence the success (or otherwise) of the sit-stand workstation intervention in influencing sitting and physical activity. Specifically, interviews with participants will explore processes that influence use of sitstand workstations, such as experiences of standing, relationships with other employees and working identities, and will last approximately 60 minutes. These will take place with 12 participants at two time points; 7-months and 12-months following the installation of sit-stand workstations. Interviews with key stakeholders and decision makers in the implementation process from the participating organisations, will elicit understanding of the feasibility, acceptability and sustainability of the sit-stand workstation intervention. Interviewees will include staff working in employee wellbeing, human resources, IT, health and safety, and facilities, as well as estates personnel and senior directors. Approximately ten stakeholders from each organisation will engage in 45 minute telephone interviews, within two months of sit-stand workstations being installed.

\section{Sample size}

A formal power analysis to determine sample size was not conducted as the threshold for meaningful reductions in sitting is unknown. The proposed sample size of the present study $(\mathrm{N}=30)$ is similar to previous pilot studies utilising sit-stand workstations in office environments that have reported significant changes in outcomes related to sitting, standing and stepping e.g. $[35,49,50]$. 


\section{Data analysis}

\section{ActivPAL3 $^{\text {TM }}$}

The monitor records the exact duration of each bout of sitting (or lying), standing, and stepping. Data will be downloaded and processed further using ActivPAL ${ }^{\text {тм }}$ proprietary software, PAL Analysis 7.

\section{ActiGraph GT3X+}

Activity counts will be recorded at 1-second intervals. Data will be downloaded and processed using proprietary software, ActiLife 6. Time spent in light, moderate and vigorous activity will be calculated by classifying vector magnitude counts according to the cut-points developed by Troiano; light activity is considered 101 to 2019 counts per min, moderate activity is considered 2020 - 5998 counts per min, and vigorous activity $\geq 5999$ counts per min. These cut-points have been validated in the general adult population [51].

\section{Non-wear time criteria}

ActiGraph GT3X+ non-wear criteria will be developed based on acceptable standards and examination of the data, as different criteria have been shown to impact the final sample size and outcome variables [52]. By comparing the ActiGraph GT3X+ to the ActivPAL3 ${ }^{\text {TM }}$ output, it will be possible to ascertain which criteria are most accurate for the data set.

\section{Qualitative data analysis}

Brief ethnographic field notes will be recorded throughout the day during the participant observation phases, with expanded field notes being written at the end of each day of participant observation. All interviews will be recorded using an Olympus LS-11 Dictaphone and transcribed ad verbatim. Qualitative data will be analysed using thematic content analysis via NVivo 10 software. Thematic analysis reports detail-rich data by identifying, analysing and interpreting patterns within the data [53]. Analysis will initially involve reading and re-reading the field notes and transcripts to become fully immersed in "the details and specifics of the data" to allow unearthing of patterns, themes and interrelationships ([54] p.362). Data will then be coded before searching for, identifying, reviewing and defining themes [55].

\section{Statistical methods}

Two-way repeated measures ANCOVA will be conducted using IBM SPSS Statistics 20 to compare the outcome measures between study arms and across the intervention (between data collection time points). Relevant covariates will be controlled for, including body mass index.

\section{Discussion}

Given the accumulating evidence of the health risks of prolonged sitting [2-8], and the epidemiological evidence that illustrates the high occurrence of prolonged sitting in office-based workplaces [18-20], high quality intervention studies are necessary to provide an evidence-base of 'what works' to reduce prolonged sitting and increase activity in the workplace. It is not only necessary to understand whether an intervention is effective, but also to understand why an intervention is, or is not, feasible, effective or sustainable. Accordingly, the contiguous systematic and rigorous process evaluation integral to this study represents an innovation within this area of research. Eliciting understanding of the social processes that underpin use of sit-stand workstations and physical activity within the workplace is crucial for understanding how to influence behaviour. A strength of the present study is that it will create a comprehensive picture of total physical activity using two objective measures; these will capture sitting, standing, and light, moderate and vigorous physical activity, alongside an activity diary, that will provide contextual information. The latter will include the type and purpose of the activities and the inactive behaviours undertaken. An understanding of the influence of the sit-stand workstation intervention on physical activity during non-work hours will also be permissible, as physical activity will be measured over the course of the entire day. A further strength is the longterm nature of the evaluation, which permits assessment of the sustainability of sit-stand workstation approaches for reducing prolonged sitting and increasing physical activity in the workplace [35]. The findings of this pilot study will provide evidence to guide future research, as well as the development of guidelines and policy to optimise the promotion of workplace health and wellbeing.

\section{Abbreviations \\ CG: Control group; PHE: Public Health England; RCT: Randomised control trail; SS-MC: Multi-component sit-stand workstation intervention; SS-WO: Sit-stand workstation only.}

\section{Competing interests}

The authors declare that they have no competing interests.

\section{Authors' contributions}

$\mathrm{JH}$ conceived of the study, participated in the design of the study and drafted the manuscript. LM participated in the design of the study and helped draft the manuscript. TK participated in the design of the study and critically revised the manuscript. AM participated in the design of the study and critically revised the manuscript. All authors read and approved the final manuscript.

\section{Authors' information}

$J \mathrm{H}$ is a PhD researcher in physical activity and health. LM is Deputy Director of the Brunel Centre for Health and Wellbeing and Senior Lecturer in Sport, Health and Social Science. TK is Theme Leader, Social Science and Health and Professor of Sport and Social Science.AM is Professor of Applied Physiology. 


\section{Acknowledgements}

This research is supported by funding from Macmillan Cancer Support. Jo Foster and Jenna Stockwell from Macmillan have contributed to the design of the study and production of materials. Sit-stand workstations will be provided by Ergotron Inc. (www.ergotron.com). James Winslade and Carrie Schmitz from Ergotron will provide ongoing logistical and ergonomic expertise. We would like to acknowledge the continuing support of key personnel and study participants at Macmillan Cancer Support and Public Health England, and the provision of study sites from both organisations.

\section{Author details}

'Division of Sport, Health and Exercise Sciences, Department of Life Sciences, Brunel University London, London, Middlesex UB8 3PH, England, UK. ${ }^{2}$ Social Sciences and Health Theme, Brunel University London, London, UK.

\section{Received: 30 January 2015 Accepted: 5 February 2015}

\section{Published online: 15 February 2015}

\section{References}

1. Pate RR, O'Neill JR, Lobelo F. The evolving definition of "sedentary". Exerc Sport Sci Rev. 2008;36(4):173-8.

2. van der Ploeg HP, Chey T, Korda RJ, Banks E, Bauman A. Sitting time and all-cause mortality risk in 222497 Australian adults. Arch Intern Med. 2012;172(6):494.

3. Thorp AA, Owen N, Neuhaus M, Dunstan DW. Sedentary behaviors and subsequent health outcomes in adults: a systematic review of longitudinal studies, 1996-2011. Am J Prev Med. 2011;41(2):207-15.

4. Proper KI, Singh AS, Van Mechelen W, Chinapaw MJ. Sedentary behaviors and health outcomes among adults: a systematic review of prospective studies. Am J Prev Med. 2011;40(2):174-82.

5. Dunstan DW, Salmon J, Owen N, Kingwell BA, Larsen R, Healy GN, et al. Breaking up prolonged sitting reduces postprandial glucose and insulin responses. Diabetes Care. 2012;35(5):976-83.

6. Healy GN, Dunstan DW, Salmon J, Cerin E, Shaw JE, Zimmet PZ, et al. Breaks in sedentary time: beneficial associations with metabolic risk. Diabetes Care. 2008;31(4):661-6.

7. Tremblay MS, Colley RC, Saunders TJ, Healy GN, Owen N. Physiological and health implications of a sedentary lifestyle. Appl Physiol Nutr Metab. 2010;35(6):725-40.

8. Sjögren P, Fisher R, Kallings L, Svenson U, Roos G, Hellénius M-L. Stand up for health-avoiding sedentary behaviour might lengthen your telomeres: secondary outcomes from a physical activity RCT in older people. Br J Sports Med. 2014;48(19):1407-9.

9. Shammas MA. Telomeres, lifestyle, cancer, and aging. Curr Opin Clin Nutr Metab Care. 2011;14(1):28-34.

10. Hamilton MT, Healy GN, Dunstan DW, Zderic TW, Owen N. Too little exercise and too much sitting: inactivity physiology and the need for new recommendations on sedentary behavior. Curr Cardiovasc Risk Rep. 2008;2(4):292-8

11. Active SAS. Start active, stay active: a report on physical activity for health from the four home countries' chief medical officers 2011. United Kingdom: Department of Health; 2011. Available from https://www.gov.uk/ government/publications/start-active-stay-active-a-report-on-physicalactivity-from-the-four-home-countries-chief-medical-officers.

12. Caspersen CJ, Powell KE, Christenson GM. Physical activity, exercise, and physical fitness: definitions and distinctions for health-related research. Public Health Rep. 1985;100(2):126.

13. Priest N, Armstrong R, Doyle J, Waters E. Policy interventions implemented through sporting organisations for promoting healthy behaviour change. Cochrane Database Syst Rev. 2008; Issue 3. Art. No.: CD004809. doi:10.1002/14651858.CD004809.pub3.

14. Owen N, Sugiyama T, Eakin EE, Gardiner PA, Tremblay MS, Sallis JF. Adults' sedentary behavior determinants and interventions. Am J Prev Med. 2011;41(2):189.

15. Ford ES, Merritt RK, Heath GW, Powell KE, Washburn RA, Kriska A, et al. Physical activity behaviors in lower and higher socioeconomic status populations. Am J Epidemiol. 1991;133(12):1246-56.

16. Marshall SJ, Jones DA, Ainsworth BE, Reis JP, Levy SS, Macera CA. Race/ ethnicity, social class, and leisure-time physical inactivity. Med Sci Sports Exerc. 2007;39(1):44-51.
17. Statistics ON. Labour Market Statistics, August 2014 release. 2014.

18. Batt ME. Physical activity interventions in the workplace: the rationale and future direction for workplace wellness. Br J Sports Med. 2009;43(1):47-8.

19. Parry $S$, Straker $L$. The contribution of office work to sedentary behaviour associated risk. BMC Public Health. 2013;13(1):296-96.

20. Ryde GC, Brown HE, Gilson ND, Brown W. Are we chained to our desks? Describing desk-based sitting using a novel measure of occupational sitting. J Phys Act Health. 2013;11(7):1318-23.

21. Torbeyns T, Bailey S, Bos I, Meeusen R. Active workstations to fight sedentary behaviour. Sports Med. 2014:44(9):1261-73.

22. Neuhaus M, Eakin E, Straker L, Owen N, Dunstan D, Reid N, et al. Reducing occupational sedentary time: a systematic review and meta-analysis of evidence on activity-permissive workstations. Obes Rev. 2014;15(10):822-38.

23. Buckley JP, Mellor DD, Morris M, Joseph F. Standing-based office work shows encouraging signs of attenuating post-prandial glycaemic excursion. Occup Environ Med. 2014;0emed-2013-101823.

24. Karakolis T, Callaghan JP. The impact of sit-stand office workstations on worker discomfort and productivity: a review. Appl Ergon. 2014;45(3):799-806.

25. Glanz K, Rimer BK, Viswanath K. Health behavior and health education: theory, research, and practice. San Francisco, Calif: Jossey-Bass; 2008.

26. Neuhaus M, Healy GN, Dunstan DW, Owen N, Eakin EG. Workplace sitting and height-adjustable workstations: a randomized controlled trial. Am J Prev Med. 2014;46(1):30-40.

27. Higgins JP, Green S: Cochrane handbook for systematic reviews of interventions. Version 5.1.0 (updated March 2011). The Cochrane Collaboration, 2011. Available from www.cochrane-handbook.org.

28. Wijk K, Mathiassen SE. Explicit and implicit theories of change when designing and implementing preventive ergonomics interventions-a systematic literature review. Scand J Work Environ Health. 2011;37(5):363-75.

29. Tudor-Locke C, Schuna J, Frensham L, Proenca M. Changing the way we work: elevating energy expenditure with workstation alternatives. Int J Obes. 2013;38(6):755-65.

30. (EPOC). EPaOoC: what study designs should be included in an EPOC review and what should they be called? In: EPOC resources for review authors. Oslo: Norwegian Knowledge Centre for the Health Services; 2014.

31. Burton J, World Health Organization. WHO healthy workplace framework and model: background and supporting literature and practices. 2010.

32. Organization WH. Global recommendations on physical activity for health. Geneva: World Health Organization; 2010. p. 8-10.

33. Sallis JF, Cervero RB, Ascher W, Henderson KA, Kraft MK, Kerr J. An ecological approach to creating active living communities. Annu Rev Public Health. 2006;27:297-322

34. Dunstan DW, Lawler S, Healy GN, Wiesner G, Eakin EG, Neuhaus M, et al. Reducing office workers' sitting time: rationale and study design for the Stand Up Victoria cluster randomized trial. BMC Public Health. 2013;13(1):1057-57.

35. Healy GN, Fjeldsoe BS, Dunstan DW, Eakin EG, Lamontagne AD, Owen N, et al. Reducing sitting time in office workers: short-term efficacy of a multicomponent intervention. Prev Med. 2013;57(1):43-8.

36. Michie S, Abraham C, Whittington C, McAteer J, Gupta S. Effective techniques in healthy eating and physical activity interventions: a meta-regression. Health Psychol. 2009;28(6):690.

37. Michie S, Ashford S, Sniehotta FF, Dombrowski SU, Bishop A, French DP. A refined taxonomy of behaviour change techniques to help people change their physical activity and healthy eating behaviours: the CALO-RE taxonomy. Psychol Health. 2011;26(11):1479-98.

38. Craig P, Dieppe P, Macintyre S, Michie S, Nazareth I, Petticrew M. Developing and evaluating complex interventions: the new Medical Research Council guidance. BMJ Br Med J. 2008;337:a1655.

39. Miller W, Rollnick S. Motivational interviewing: preparing people for change. New York: Guilford; 2002.

40. Cavill N, Roberts K, Rutter H. Standard evaluation framework for physical activity interventions. Nat Obes Observ 2012.

41. Lyden K, Kozey Keadle SL, Staudenmayer JW, Freedson PS. Validity of two wearable monitors to estimate breaks from sedentary time. Med Sci Sports Exerc. 2012;44(11):2243-52.

42. Kozey-Keadle S, Libertine A, Lyden K, Staudenmayer J, Freedson PS. Validation of wearable monitors for assessing sedentary behavior. Med Sci Sports Exerc. 2011;43(8):1561-7. 
43. Ozemek C, Kirschner MM, Wilkerson BS, Byun W, Kaminsky LA. Intermonitor reliability of the GT3X+ accelerometer at hip, wrist and ankle sites during activities of daily living. Physiol Meas. 2014;35(2):129.

44. O'Cathain A, Goode J, Drabble SJ, Thomas KJ, Rudolph A, Hewison J. Getting added value from using qualitative research with randomized controlled trials: a qualitative interview study. Trials. 2014;15(1):215.

45. Oakley A, Strange V, Bonell C, Allen E, Stephenson J. Process evaluation in randomised controlled trials of complex interventions. BMJ. 2006;332(7538):413-6.

46. Anhøj J, Møldrup C. Feasibility of collecting diary data from asthma patients through mobile phones and SMS (short message service): response rate analysis and focus group evaluation from a pilot study. J Med Internet Res. 2004;6(4):e42.

47. Rosen M. Coming to terms with the field: understanding and doing organizational ethnography. J Manag Stud. 1991;28(1):1-24.

48. Warren C, Karner T. The interview. Discovering qualitative methods: field research, interviews and analysis. Los Angeles: Roxbury; 2005. p. 115-35.

49. Alkhajah TA, Reeves MM, Eakin EG, Winkler EAH, Owen N, Healy GN. Sit-stand workstations: a pilot intervention to reduce office sitting time. Am J Prev Med. 2012;43(3):298.

50. Dutta N, Koepp GA, Stovitz SD, Levine JA, Pereira MA. Using sit-stand workstations to decrease sedentary time in office workers: a randomized crossover trial. Int J Environ Res Public Health. 2014;11(7):6653-65.

51. Troiano RP, Berrigan D, Dodd KW, Masse LC, Tilert T, McDowell M. Physical activity in the United States measured by accelerometer. Med Sci Sports Exerc. 2008;40(1):181.

52. Masse L, Fuemmeler BF, Anderson C, Matthews CE, Trost SG, Catellier D, et al. Accelerometer data reduction: a comparison of four reduction algorithms on select outcome variables. Med Sci Sports Exerc. 2005;37(11 Supplement):S544-54

53. Braun V, Clarke V. Using thematic analysis in psychology. Qual Res Psychol. 2006;3(2):77-101.

54. Johnson RRB, Christensen LB. Educational research: quantitative, qualitative and mixed approaches. Thousand Oaks, CA: Sage Publications; 2010.

55. Sparkes AC, Smith B. Qualitative research methods in sport, exercise and health: from process to product: Routledge. 2013.

\section{Submit your next manuscript to BioMed Central and take full advantage of:}

- Convenient online submission

- Thorough peer review

- No space constraints or color figure charges

- Immediate publication on acceptance

- Inclusion in PubMed, CAS, Scopus and Google Scholar

- Research which is freely available for redistribution 